\title{
Acute pancreatitis during pregnancy: a case report
}

\begin{abstract}
Objective: is to endorse the management of pregnancy complicated with acute pancreatitis.

Background: Acute pancreatitis may endanger the life of mother and her baby especially in case of improper management or late diagnosis.

Case: We report a case of a woman in her third trimester of pregnancy who presented with nausea, vomiting and abdominal pain mainly in upper right abdomen. On examination, there was mild tenderness in epigastrium and right hypochondrium associated with elevated serum amylase, alanine transaminase, and aspartate transaminase. Her abdominal ultrasound showed multiple gallbladder stones. She delivered vaginally with good foetal condition. Conservative management was started during pregnancy then laparoscopic cholecystectomy was done after delivery.
\end{abstract}

Conclusion: Diagnosis of acute pancreatitis in pregnancy is challenging and conservative management remains a good option in case of mild acute pancreatitis, the surgical intervention should be kept for severe cases.

Keywords: acute, pancreatitis, gallbladder, stones, pregnancy
Volume 7 Issue $6-2018$

\author{
Ahmed Altraigey,' Mufareh Asiri² \\ 'Department of Obstetrics and Gynaecology, Benha University, \\ Egypt \\ ${ }^{2}$ Department of Obstetrics and Gynaecology,Armed forces \\ hospitals southern region, Saudi Arabia
}

Correspondence: Ahmed Altraigey, Department of Obstetrics and Gynaecology, Benha University, Kingdom of Saudi Arabia, 43-Benha, Zagazig Street, Egypt, Tel +966544854232, Email ahmed.altreigey@yahoo.com

Received: July 09, 2018 | Published: December 13, 2018

\section{Introduction}

Although acute pancreatitis is an uncommon event during pregnancy, its incidence was reported to be approximately 1 in 1000 to 1 in $10,000 .^{1,2}$ Misdiagnosis or delayed management of such cases could be serious and lead to high mortality. There are many recorded aetiologies, but commonest are the presence of biliary disease, congenital or acquired hypertriglyceridemia. Rarely, acute pancreatitis is associated with pre-eclampsia, eclampsia or HELLP syndrome.,

The incidence of acute pancreatitis is increasing proportionally with advanced gestational age. ${ }^{[1]}$ There is no significant difference regarding clinical presentations of acute pancreatitis in pregnant and non-pregnant women. The common symptoms are nausea, vomiting, anorexia, abdominal pain, dyspepsia and intolerance to fatty diet. The signs are mostly low-grade fever and tachycardia. ${ }^{4}$

The pathophysiology of pancreatitis in pregnancy remains unexplained but thought to be due to increased cholesterol secretion in the hepatic bile mostly during the second and third trimester as compared to phospholipids and bile acids leading to more saturated bile. Furthermore, fasting and postprandial gallbladder greater volumes as well as the reduced emptying volume contribute to more bile concentration. Finally, the big residual volume of concentrated bile in the slow gallbladder can lead to crystals and gallstones formation. ${ }^{1}$ Herby, we represent a case of third trimester pregnancy complicated with mild pancreatitis which was managed conservatively till uneventful delivery.

\section{Case report}

26 years old pregnant woman G4P3+0 at 37 weeks of gestation presented to emergency unit in Armed Forces Hospital Southern Region, Saudi Arabia complaining of nausea, vomiting and abdominal pain mainly in upper right abdomen radiating to the back. She noticed that her urine is dark in colour. By her past history, she is medically and surgically free. There was no history of fever, diarrhoea, constipation, vaginal bleeding or discharge. On examination, she was afebrile and not jaundiced. The abdomen was soft with lax gravid uterus. There was mild tenderness in epigastrium and right hypochondrium. Her laboratory investigations showed normal complete blood count and elevated liver enzymes. Serum amylase was markedly elevated as well as her total and direct bilirubin. The patient's ultrasound showed multiple gallbladder stones with average wall thickness and dilated common bile duct (Figure 1). There was no intra-peritoneal free fluid. Liver, kidneys and spleen were unremarkable. Also, it showed single viable foetus with cephalic presentation and normal to-date biometric and Doppler measurements.

The Diagnosis of acute pancreatitis was confirmed and patient was admitted and kept fasting (nothing per oral NPO). Antibiotics, intravenous fluids and pethidine were started. Her symptoms and signs were of regressive course and she was kept under observation following fat-free diet. Ten days later, she was started labour pains and delivered spontaneously uneventfully. She was discharged in a stable condition on the next day. A week later, endoscopic retrograde cholangio-pancreatography $(E R C P)$ Figure 2 was performed and common bile duct stones were extracted. She remained symptomatic, so laparoscopic cholecystectomy was done uneventful after two days. Patient was discharged home with multidisciplinary appointment for follow up on outpatient basis. The histopathological examination showed gallbladder mucosa with surrounding chronic inflammation and reactive congested wall which are characteristics of chronic cholecystitis.

\section{Discussion}

The diagnosis of acute pancreatitis in pregnancy, as a challenge, was based on combination of the clinical manifestations, laboratory and radiological investigations. However, treatment of acute pancreatitis in pregnancy should be carried by a multidisciplinary team and could be either conservative management or surgical intervention according to the severity of presentation, the general maternal and foetal condition. Acute pancreatitis in pregnancy is a rare and affront situation to manage due to limited evidence-based practice guidelines as the connection between acute pancreatitis and pregnancy is not well understood. Acute pancreatitis in pregnancy was defined according to the severity of symptoms as highlighted by revision of the Atlanta classification. ${ }^{5}$ 


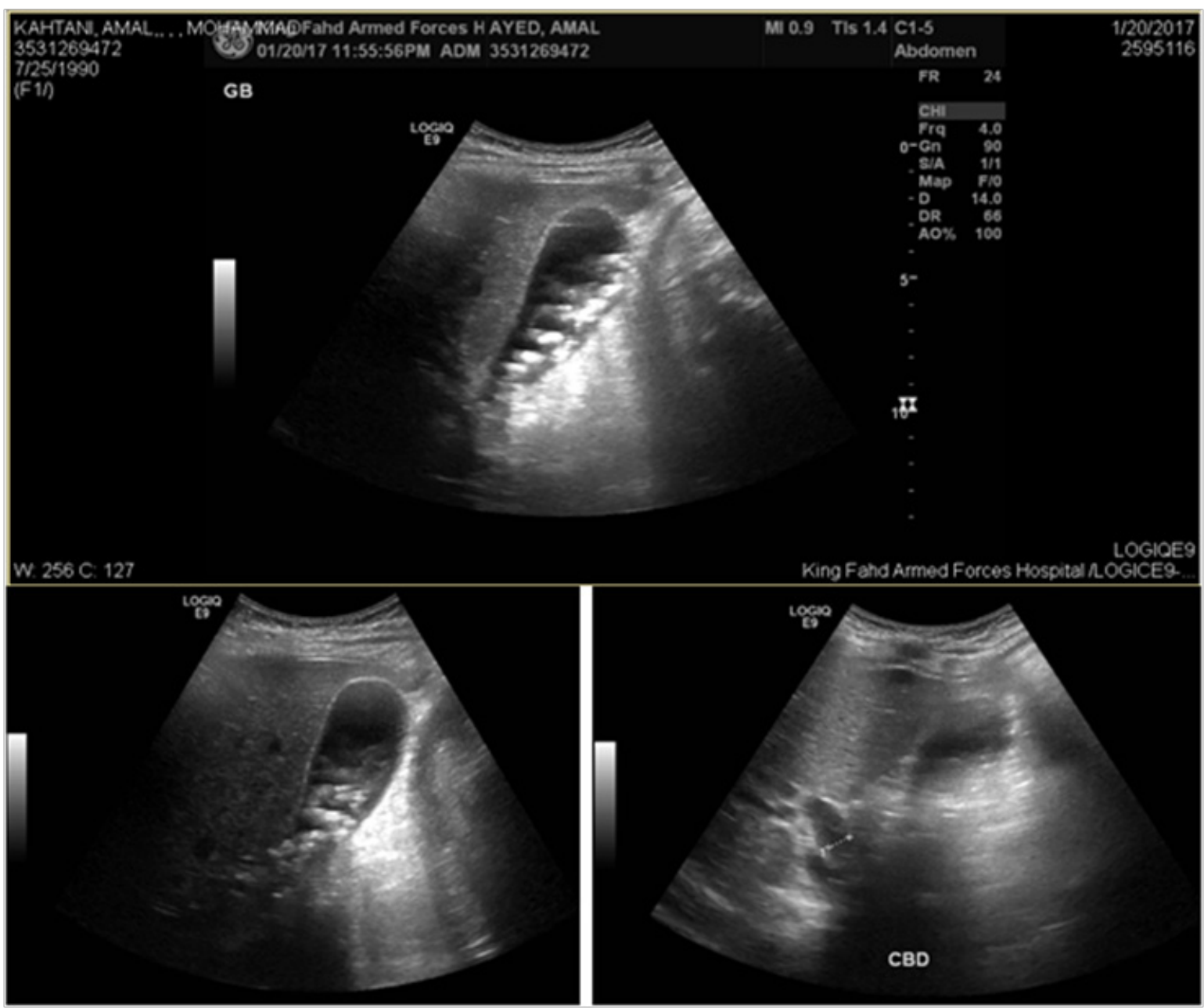

Figure I Shows gallbladder stones and dilated common bile ducts.

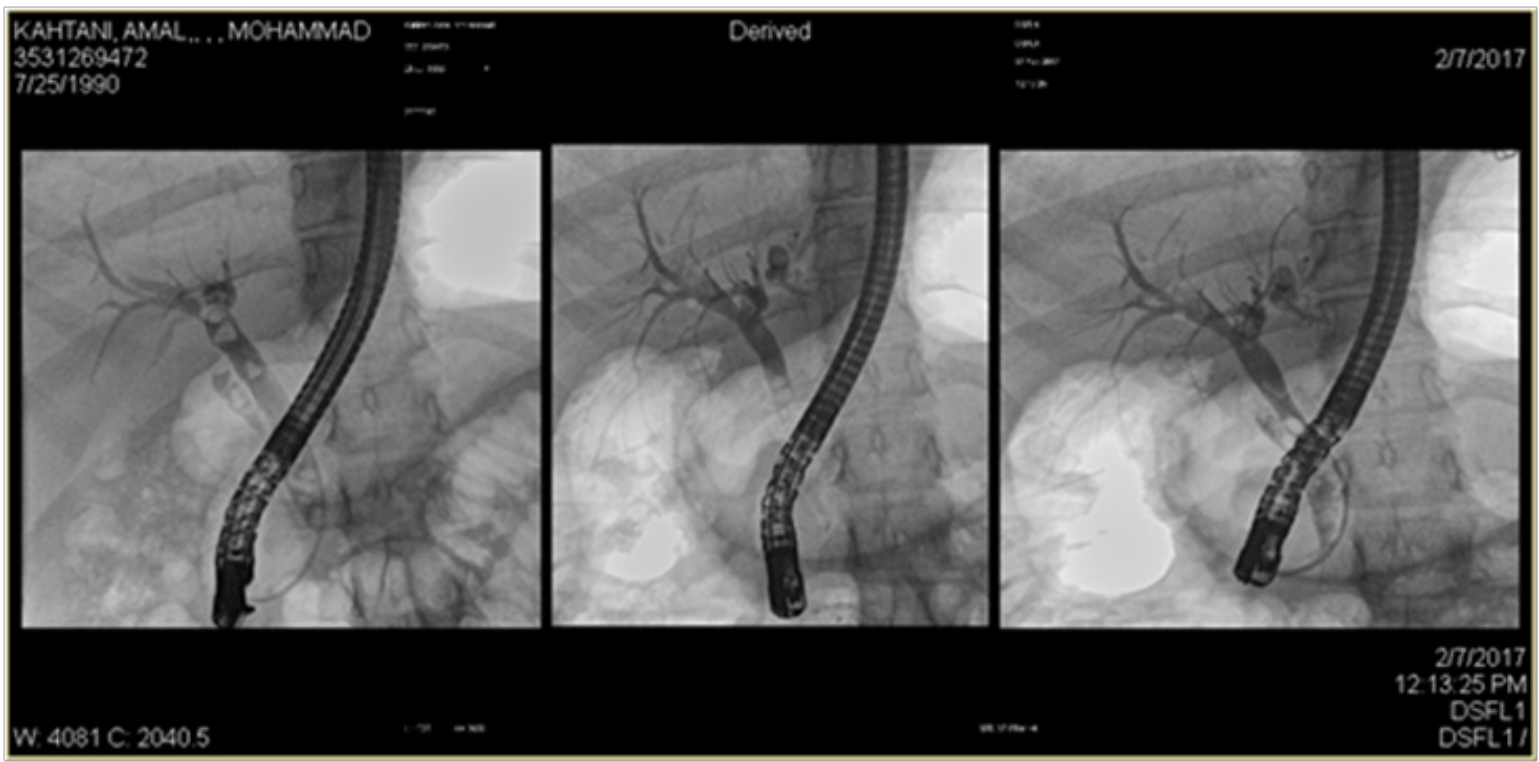

Figure 2 Shows multiple stones in the common bile ducts seen during endoscopic retrograde cholangio-pancreatography. 
From our case's history, none of such risk factors were documented except pregnancy. In pregnant women with acute pancreatitis, the commonest clinical presentations are abdominal pain $(89.47 \%)$ and vomiting $(68.42 \%))^{2}$ They may present with anorexia and fever. Bowel sounds are reduced due to a paralytic ileus and a positive Murphy's sign may be present. Associated pulmonary findings are noticed in $10 \%$ of patients. ${ }^{6}$

Most symptoms like nausea, vomiting, abdominal discomfort, or pain are frequently seen in normal pregnancy. Also, clinical evaluation of acute abdomen in pregnancy is confusing and difficult due to the anatomical displacement of abdominal organs by the gravid uterus. Some of the haematological and biochemical of acute pancreatitis are similar to normal pregnancies value, so the diagnosis of acute pancreatic needs a deeper investigations. An elevated serum amylase level has a diagnostic sensitivity of $81 \%$ and adding serum lipase increases the sensitivity of $94 \%$.

Some factors may play a role in the management of acute pancreatitis in pregnancy, including the gestational age, common bile duct dilatation, cholangitis, and the severity of acute pancreatitis. ${ }^{1}$ The use of antibiotics is controversial. The literature shows that antibiotic prophylaxis did not reduce mortality, protect against infected necrosis or minimize the frequency of surgical intervention. ${ }^{8}$ Indications for surgery during pregnancy are severe symptoms, acute cholecystitis unresponsive to medical treatment, obstructive jaundice and peritonitis. ${ }^{9}$ In the literature, conservative management is preferred for mild cases. ${ }^{10}$

In conclusion, early detection of acute pancreatitis in pregnancy and conservative management with meticulous maternal and foetal monitoring is recommended with good outcome. Surgical interventions should be limited for serious indications after full counselling by a multidisciplinary team. Due to the complexity of acute pancreatitis in pregnancy, the scientific society and committees should focus on establishing specific guidelines for the management of acute pancreatitis in pregnancy rather than to be based on expert opinions, case series or case reports.

\section{Acknowledgments}

No acknowledgements are applicable.

\section{Conflicts of interest}

There are no conflicts of interests for any author.

\section{References}

1. Pitchumoni CS, Yegneswaran B. Acute pancreatitis in pregnancy. World J Gastroenterol. 2009; 15(45):5641-5646.

2. Igbinosa O, Poddar S, Pitchumoni C. Pregnancy associated pancreatitis revisited. Clin Res Hepatol Gastroenterol. 2013;37(2):177-181.

3. Terzhumanov R, Uchikov A, Uchikova E, et al. Acute pancreatitis and pregnancy: analysis of a 10 year period of time. Akush Ginekol (Sofiia). 2004;43(7):9-12.

4. Ko CW, Beresford SA, Schulte SJ, et al. Incidence, natural history, and risk factors for biliary sludge and stones during pregnancy. Hepatology. 2005;41(2):359-365.

5. Banks PA, Bollen TL, Dervenis C, et al. Acute Pancreatitis Classification Working Group. Classification of acute pancreatitis-2012: revision of the Atlanta classification and definitions by international consensus. Gut. 2013;62(1):102-111

6. Pandey R, Jacob A, Brooks H. Acute pancreatitis in pregnancy: review of three cases and anaesthetic management. Int $J$ Obstet Anesth. 2012;21(4):360-363.

7. Ramin KD, Ramin SM, Richey SD, et al. Acute pancreatitis in pregnancy. Am J Obstet Gynecol. 1995;173(1):187-191.

8. Vishnu Priya KMN, Sheela CN, Shruti Banka, et al. Maternal and perinatal outcome of acute pancreatitis during pregnancy: a 5 year experience at a tertiary care centre. Int $J$ Reprod Contracept Obstet Gynecol. 2016;5(11):4041-4045.

9. Ducarme G, Maire F, Chatel P, et al. Acute pancreatitis during pregnancy: a review. J Perinatol. 2014;34(2):87-94.

10. Juneja SK, Gupta S, Virk SS, et al. Acute pancreatitis in pregnancy: A treatment paradigm based on our hospital experience. Int J Appl Basic Med Res. 2013;3(2):122-125. 